\title{
ROTATIONAL MOTION OF CELESTIAL BODIES
}

\section{IN THE RELATIVISTIC FRAMEWORK}

\author{
SERGEI A. KLIONER and \\ MICHAEL SOFFEL \\ Lohrmann Observatory, Dresden Technical University, 01062 Dresden, Germany \\ e-mail:klioner(soffel)@rcs.urz.tu-dresden.de
}

There are several important reasons to consider relativistic effects in rotational motion of celestial bodies. General Relativity is now recommended by the International Astronomical Union and Intemational Union of Geodesy and Geophysics as a theoretical framework for modeling of high-precision observational data. On the other hand, various geodynamical observations provide data which are widely used for testing General Relativity itself.

In Newtonian mechanics it is well known how to describe rotational motion of an extended body. In General Relativity this is a rather subtle issue. The concept of a precessing extended rigid body in general relativity encounters fundamental difficulties and cannot be introduced even in the first post-Newtonian approximation. From a practical point of view, however, the rotational motion of the Earth even at the Newtonian level is defined operationally through the time-dependence of geocentric quasi-inertial coordinates of observing sites. An analogous operational definition can be applied in general relativity. To this end, we need a set of physically adequate reference systems.

Nowadays there are two well-developed formalisms for the construction of relativistic astronomical reference systems: the Brumberg-Kopeikin formalism (see, e.g., Brumberg, 1991) and the DSX formalism (Damour, Soffel, Xu, 1991, 1992, 1993). The two reference systems needed to model the Earth rotation are the barycentric reference system of the solar system and the local geocentric reference system, where the influence of the external masses reduces to tidal effects. Each reference system is defined by the structure of its metric tensor.

In the local geocentric reference system one can derive rotational equations of motion of the extended deformable arbitrarily-shaped Earth, which take the same form as in Newtonian physics

$$
\frac{d}{d t} S^{i}=L^{i}
$$

where $S^{i}$ is the post-Newtonian spin, and $L^{i}$ is the post-Newtonian tidal torque (Damour, Soffel, Xu, 1993; Klioner, 1996). Eq. (1) is sufficient to discuss precession and nutation of the spin. To consider precession and nutation of the angular velocity and figure axis, we need a relativistic definition of the tensor of inertia and angular velocity. A variety of theoretical approaches (from restricted rigid body models (Soffel, 1994) to relativistic Tisserand-like axes of deformable Earth (Klioner, 1996)) lead to the same post-Newtonian definition of the tensor of inertia $C^{i j}$. The 
spin can be then split into a product of the tensor of inertia and the corresponding angular velocity $\omega^{i}$

$$
S^{i}=C^{i j} \omega^{j} \text {. }
$$

The definition of angular velocity of a deformable Earth is not unique already in Newtonian mechanics (Tisserand axes, principal axes of inertia, etc.). This ambiguity is aggravated in general relativity by the ambiguity in the definition of the relativistic spin $S^{i}$.

There are three main observational consequences of general relativity in Earth rotation: (a) The kinematically nonrotating geocentric reference system used in practice rotates relative to a locally inertial reference system. This results in Coriolis and centrifugal forces in the right-hand side of (1) resulting in geodetic precession ( $\sim 1.9^{\prime \prime}$ per century) and nutation (with a period of one year and an amplitude of 0.15 mas). (b) Explicit relativistic terms in $L^{i}$ produce additional periodic relativistic effects amounting to $\sim 1$ microarcsecond (Bizouard, et al., 1992). (c) The key relation of Newtonian mechanics

$$
\left(M^{i j}\right)_{\text {Newton }}=\left(\frac{1}{3} \delta^{i j} C^{s s}-C^{i j}\right)_{\text {Newton }}
$$

between the quadrupole moment of the gravitational field $M^{i j}$ and the tensor of inertia $C^{i j}$ is violated in general relativity. This means that $J_{2}$, derived from $M^{i j}$, cannot be easily related to the dynamical ellipticity $H$, which is derived from $C^{i j}$, the effect being of order of $\delta J_{2} / J_{2} \sim 10^{-9}$ (Klioner, 1997).

\section{References}

Bizouard C., Schastok, J., Soffel M.H., Souchay J.: 1992, Étude de la rotation de la Terre dans le cadre de la relativité générale: première approche. In: N. Capitaine (ed.), Journées 1992, Observatoire de Paris, 76

Brumberg V.A.: 1991, Essential Relativistic Celestial Mechanics, Adam Hilger, Bristol

Damour T., Soffel M., Xu Ch.,: 1991, Phys.Rev.D, 43, 3273

Damour T., Soffel M., Xu Ch.,: 1992, Phys.Rev. D, 45, 1017

Damour T., Soffel M., Xu Ch.,: 1993, Phys.Rev. D, 47, 3124

Klioner, S.A.: 1996, Angular Velocity of Rotation of Extended Bodies in General Relativity. In: S. Ferraz-Mello, B. Morando, J.E. Arlot (eds.), Dynamics, ephemerides and astrometry in the solar system, Kluwer, Dordrecht, 309

Klioner, S.A.: 1997, On the problem of post-Newtonian Rotational Motion In: I.M. Wytrzysczak, J.H. Lieske, R.A. Feldman (eds.), Dynamics and Astrometry of Natural and Artificial Celestial Bodies, Kluwer, Dordrecht, 383

Soffel, M.: 1994, The problem of rotational motion and rigid bodies in the post-Newtonian framework, unpublished notes 